Nouvelles perspectives en sciences sociales

Revue internationale de systémique complexe et d'études relationnelles

\title{
Une sociologie cosmopolite : esquisse d'un changement paradigmatique
}

\section{Ulrich Beck}

Volume 8, numéro 1, novembre 2012

URI : https://id.erudit.org/iderudit/1013921ar

DOI : https://doi.org/10.7202/1013921ar

Aller au sommaire du numéro

Éditeur(s)

Prise de parole

ISSN

1712-8307 (imprimé)

1918-7475 (numérique)

Découvrir la revue

Citer cet article

Beck, U. (2012). Une sociologie cosmopolite : esquisse d'un changement paradigmatique. Nouvelles perspectives en sciences sociales, 8(1), 161-190.

https://doi.org/10.7202/1013921ar d'utilisation que vous pouvez consulter en ligne. 


\title{
Une sociologie cosmopolite : esquisse d'un changement paradigmatique ${ }^{1}$
}

\author{
ULRICH BECK \\ Institute for Sociology \\ Ludwig Maximilian University of Munich
}

\begin{abstract}
u cours des dernières années, on a assisté à la renaissance du $\mathbf{A}_{\text {cosmopolitisme, mais le sens sociologique de cette renais- }}$ sance n'est pas aisément saisissable. Comme j'espère le montrer, le fait est que le cosmopolitisme a cessé d'être simplement une idée et un idéal controversés; quand ce ne serait que sous la forme d'une distorsion, il a quitté les sphères des châteaux philosophiques stratosphériques et il est entré dans la réalité. En effet, il est devenu le trait caractéristique d'une ère nouvelle, l'ère de la modernité réfléchie, dans laquelle le regard national, qui opère avec une hypothèse non problématisée de nette correspondance entre nation, territoire, société, politique et culture, laisse échapper la réalité. Ainsi, le mot " cosmopolite » devient indispensable pour décrire une situation dans laquelle " humanité » et « monde " sont des catégories sociales, politiques et morales non seulement auxquelles on peut penser pour dépeindre la condition humaine, mais aussi qui sont inévitables dès lors qu'on veut le faire. C'est

$1 \quad$ Traduit de l'anglais par Simon Laflamme. Le texte a été initialement publié sous le titre "Cosmopolitan Sociology : Outline of a Paradigm Shift " dans The Ashgate Research Companion to Cosmopolitanism (Maria Rovisco et Magdalena Nowicka (dir.), Surrey, Ashgate Publishing Limited, 2011, p. 17-32).
\end{abstract}


la raison pour laquelle un monde qui est devenu cosmopolite réclame avec urgence un nouveau point de vue, la perspective cosmopolite, à partir duquel on peut saisir les réalités sociales et politiques dans lesquelles nous vivons et agissons. La perspective cosmopolite est à la fois le présupposé et le résultat d'une reconfiguration conceptuelle de nos modes de perception.

Pour que cette conceptualisation se réalise en des termes des sciences sociales, il est nécessaire de déplacer le concept de cosmopolitisme pour qu'il aille de sa tête, qui est philosophique, vers ses pieds, qui appartiennent aux sciences sociales².

D'abord, je remettrai en question l'une des plus puissantes convictions relatives à la société et à la politique et qui lient les acteurs sociaux et les spécialistes des sciences sociales : le nationalisme méthodologique. Le nationalisme méthodologique traite comme équivalentes la société moderne et la société organisée dans le territoire limité de l'État-nation.

Deuxièmement, je proposerai de marquer une distinction essentielle entre cosmopolitisme, dans un sens philosophique normal, et cosmopolitisation, comme programme de recherche en sciences sociales.

Troisièmement, j'illustrerai ce changement de paradigme en reconfigurant le concept d'inégalité sociale transnationale et, quatrièmement, celui de risque mondial. Cinquièmement, et finalement, je reprendai la question : que signifie « cosmopolitisme " méthodologique?

\section{Critique du nationalisme méthodologique}

Le nationalisme méthodologique présuppose que la nation, l'État et la société sont les formes sociales et politiques naturelles du monde moderne. Il suppose que l'humanité est naturellement divisée en un nombre limité de nations qui, de l'intérieur, s'organisent elles-mêmes en États-nations et, à l'extérieur, établissent des frontières pour se distinguer des autres États-nations. Cela va même plus loin : cette délimitation extérieure, aussi bien que la

2 Ulrich Beck et Edgar Grande, Cosmopolitan Europe, Cambridge (UK) / Malden (MA), Polity Press, 2007. 
compétition entre les États-nations, représente la catégorie la plus fondamentale de l'organisation politique. En effet, la position des sciences sociales est enracinée dans le concept d'État-nation. Il s'agit d'une perspective de l'État-nation sur la société et la politique, la loi, la justice et l'histoire qui gouverne l'imagination sociologique. Et c'est exactement ce nationalisme méthodologique qui empêche les sciences sociales de comprendre le cœur des dynamiques de la modernisation et de la mondialisation, aussi bien au passé qu'au présent : le résultat involontaire de la radicalisation de la modernité est l'atténuation du pouvoir des États occidentaux, ce qui est brutalement en contraste avec l'autonomisation qu'ils ont connue avant et pendant la vague de mondialisation du XIX ${ }^{\mathrm{e}}$ siècle $^{3}$.

Quand les acteurs sociaux souscrivent à cette croyance, je parle d'une perspective nationale; quand cette croyance détermine l'approche de l'observateur en sciences sociales, je parle d'un "nationalisme méthodologique ». La distinction entre la perspective de l'acteur social et celle du spécialiste des sciences sociales est cruciale parce que la connexion entre les deux est historique, et non logique. Cette connexion historique - entre acteurs sociaux et spécialiste des sciences sociales - à elle seule met en évidence l'axiomatique du nationalisme méthodologique. Et le nationalisme méthodologique n'est pas un problème superficiel ou une erreur mineure. Il implique à la fois les routines de la collecte et de la production de données et les concepts de base de la sociologie moderne et de la science politique - des concepts comme société, inégalité sociale, État, démocratie, communautés imaginaires.

Il est évident que, au XIX ${ }^{\mathrm{e}}$ siècle, la sociologie européenne a été formulée à l'intérieur d'un paradigme nationaliste et que les sentiments cosmopolites ont été étouffés par les horreurs des grandes guerres. Dans le nationalisme méthodologique d'Émile Durkheim, la fraternité est devenue solidarité et intégration

3 Ulrich Beck, "The Cosmopolitan Condition: Why Methodological Nationalism Fails ", Theory, Culture and Society, vol. 24, $\mathrm{n}^{\text {os }} 7-8,2007$, p. 286-290. 
nationale. Durkheim avait, bien sûr, à l'esprit l'intégration de la société nationale (la France) sans même la mentionner, la nommer ou penser à elle (mais il est vrai aussi que, en même temps, Durkheim référait au cosmopolitisme comme à une possibilité). En fait, ne pas employer l'adjectif «national » comme langage universel ne falsifie pas le nationalisme méthodologique; cela peut même parfois constituer la preuve de son existence. La sociologie de Max Weber impliquait une étude comparative des religions du monde dans leur rapport à l'économie; mais l'inspiration politique de sa sociologie (politique) était nationalistique (nationalistic). En Amérique du Nord, l'existence du même paradigme national est manifeste. Bien sûr, Talcott Parsons a adopté une approche sociologique comparative, et il était un élève de la pensée sociale européenne; mais ses préoccupations et son approche sociologiques étaient américaines. En majeure partie, la sociologie classique aujourd'hui étudie la "société nationale " sous le parapluie de la "société ». On ne doit pas oublier que la sociologie classique était le produit de luttes nationales : la guerre franco-allemande de 1870 et la Première Guerre mondiale au début du XXe siècle ${ }^{4}$.

La critique du nationalisme méthodologique ne doit pas être confondue avec la thèse selon laquelle la fin de l'État-nation est arrivée. On ne critique pas l'individualisme méthodologique en proclamant la fin de l'individu. Les États-nations (comme le montrent toutes les recherches) continueront de prospérer ou seront transformés en État transnationaux (par exemple l'Union Européenne). Le point décisif est que l'organisation nationale, comme principe structurant de l'action sociétale et politique, ne peut plus servir en tant que point de référence orientant l'observateur en sciences sociales. On ne peut même plus comprendre la tendance à la re-nationalisation ou à la ré-ethnicisation en Europe de l'Ouest ou de l'Est et dans d'autres parties du monde sans une perspective cosmopolite. En ce sens, les sciences sociales

$4 \quad$ Ibid.; Ulrich Beck et Edgar Grande, "Varieties of Second Modernity: The "Cosmopolitan Turn" in Social and Political Theory and Research ", British Journal of Sociology, vol. 61, n 3, 2010, p. 409-443. 
ne peuvent répondre adéquatement au défi de la mondialisation que si elles parviennent à dépasser le nationalisme méthodologique et à poser empiriquement et théoriquement des questions fondamentales dans des champs spécialisés de recherche, et ainsi à établir les fondements d'une science sociale cosmopolite nouvellement formulées.

\section{La distinction cruciale entre le cosmopolitisme normatif et la cosmopolitisation empirico-analytique}

On peut distinguer trois phases dans la manière dont le mot "mondialisation " (globalization) a été employé dans les sciences sociales : premièrement, le déni; deuxièmement, l'affinement conceptuel et la recherche empirique; troisièmement, la « cosmopolitisation ». Le déni initial n'a plus cours parce que l'affinement théorique et empirique a révélé par ses réalisations un nouveau paysage $^{6}$. Parmi ses traits dominants de ce paysage nouveau, il y a l'interconnectivité, ce qui signifie la dépendance et l'interdépendance des personnes tout autour du globe. Virtuellement, la totalité des expériences et des pratiques humaines est de quelque façon influencée par l'écrasante interconnectivité du monde. (Ce

5 Ulrich Beck et Edgar Grande, "Varieties of Second Modernity: The "Cosmopolitan Turn" in Social and Political Theory and Research ", op. cit.; Ulrich Beck et Natan Sznaider, "Unpacking Cosmopolitanism for the Social Sciences: A Research Agenda ", British Journal of Sociology, vol. 57, n 1, 2006, p. 1-23; Anja, Weiß, «Vergleiche jenseits des Nationalstaats. Methodologischer Kosmopolitismus in der soziologischen Forschung über hochqualifizierte Migration ", Soziale Welt, vol. 61, nos 3-4, 2010, p. 295-311.

6 Voir, entre autres : Zygmunt Bauman, Globalization: The Human Consequences, New York, Columbia University Press, 1998; Ulrich Beck, What Is Globalization?, Cambridge (UK) / Malden (MA), Polity Press, 2000; Marianne Beisheim, Sabine Dreher, Gregor Walter, Bernhard Zangl et Michael Zürn, Im Zeitalter der Globalisierung? Thesen und Debatten zur gesellschaftichen und politischen Denationalisierung, Baden-Baden, Nomos Verlagsgesellschaft, 1999; David Held, Anthony McGrew, David Goldblatt et Jonathan Perraton, Global Transformations: Politics, Economics and Culture, Cambridge (UK) / Stanford (CA), Polity Press / Stanford University Press, 1999; Shalini Randeria, Shalini, Local Refractions of Global Governance: Legal Plurality, International Institutions, the Post-Colonial State and NGOs in India, Habilitation, Free University of Berlin, 2001; Saskia Sassen, "Globalization or Denationalization? ", vol. $10, \mathrm{n}^{\circ} 1,2003$, p. 1-22. 
propos ne doit pas être confondu avec les théories du système du monde et de la dépendance.)

La troisième phase dévoile la principale conséquence, non vue, non voulue, de cette interconnectivité globale : la fin de l'" autre global ». L'autre global est ici, parmi nous. Bien sûr, la controverse entourant les caricatures de Mohamed en donne un exemple remarquable ${ }^{7}$. Les caricatures incarnent l'intersection du global et du local en matière de communication et de conflit à l'ère du digital et en ce qui a trait à la politique. Lautre global est et demeure parmi nous, ne peut plus être exclu. $\mathrm{Ou}$, pour le dire de cette façon : même si le gouvernement danois décidait d'exclure tous les musulmans danois ou non danois, cela ne résoudrait pas le conflit; en fait, c'est le contraire qui clairement surviendrait : un outrage global et une explosion de conflits. Et c'est là précisément ma position : demander de distinguer clairement entre le cosmopolitisme philosophique et la cosmopolitisation en sciences sociales. Le cosmopolitisme dans le sens philosophique d'Emmanuel Kant signifie quelque chose d'actif, une tâche, un choix conscient et volontaire, clairement l'affaire d'une élite, une consigne qui va de haut en bas ${ }^{8}$. Mais aujourd'hui, en réalité, une cosmopolitisation "banale ", " coercitive " et "impure " se déploie, non voulue, non vue, très puissante et provocante sous la surface ou derrière la façade des étiquettes, des juridictions, des espaces nationaux persistants, depuis le sommet de la société jusque dans la vie quotidienne des familles, dans les situations de travail et dans les biographies individuelles - alors même qu'on continue de brandir les drapeaux nationaux et que les attitudes, les identités et les consciences nationales sont fortement réaffirmées. Ainsi, les nouveaux paysages volcaniques des "sociétés "

$7 \quad$ Kunelius, Risto, Elisabeth Eide, Oliver Hahn et Roland Schroeder (dir.), Reading the Mohammed Cartoons Controversy: An International Analysis of Press Discourses on Free Speech and Political Spin, Bochum/Freiburg, Projektverlag, 2007.

8 Immanuel Kant, "Idea for a Universal History with a Cosmopolitan Purpose ", dans Perpetual Peace and Other Essays on Politics, History and Morals, Indianapolis, Hackett, 1983, p. 29-39; Immanuel Kant, "Toward Perpetual Peace: A Philosophical Project ", dans Practical Philosophy, Cambridge, Cambridge University Press, 1996, p. 311-351. 
doivent être reconfigurés aux niveaux macro aussi bien que micro, et horizontalement à travers les grands champs que sont la communication, les interactions et les pratiques sociales et politiques ${ }^{9}$.

Avec les changements climatiques, la plupart des impulsions importantes pour les transformations sociales et économiques du nouveau siècle ne valent pas de façon différentielle ou exclusive pour certains groupes ou certaines nations. Pensons à ce qui suit : la financiarisation et le libre échange mondiaux, la déterritorialisation des entreprises et la production transnationale, l'usage mondialisé du travail, la concurrence et les conflits de classes, la concertation mondiale et la formulation de règles (administrées par le FMI, etc.), la communication par Internet et le cyberespace, la manipulation bioscientifique de formes de vie (incluant graduellement des corps humains) mondialement orchestrée, les risques mondiaux de toutes sortes (crises financières, terrorisme, SIDA, grippe porcine, etc.), les ajustements démographiques transnationaux (migration de la main-d'œuvre, des époux, des enfants), les arts et le divertissement cosmopolitisés, et, enfin, et non les moindres, les guerres régionales mondialement financées et gérées. Il n'y a pas de hiérarchies, de séquences ou de sélectivités systémiques permanentes par lesquelles divers groupes ou nations - que ce soit à différents niveaux de développement, dans

9 Pour une discussion sur la distinction entre - " pur »- cosmopolitisme et - "impure "-cosmopolitisation, voir aussi : Ulrich Beck et Edgar Grande, "Varieties of Second Modernity: The "Cosmopolitan Turn" in Social and Political Theory and Research ", op. cit.; Craig Calhoun, "Beck, Asia and Second Modernity », British Journal of Sociology, vol. 61, n³, 2010, p. 597 619; Kyung-Sup Chang, "The Second Modern Condition? Compressed Modernity as Internalized Reflexive Cosmopolitanism ", British Journal of Sociology, vol. 61, n 3, 2010, p. 444-464; Paul Gilroy, "Planetarity and Cosmopolitics ", British Journal of Sociology, vol. 61, n 3, 2010, p. 620-626; Daniel Levy, "Recursive Cosmopolitization: Argentina and the Global Human Rights Regime ", British Journal of Sociology, vol. 61, n 3, 2010, p. 579-596; Sarat Maharaj, "Small Change of the Universal: Beyond Modernity? ", British Journal of Sociology, vol. 61, n 3, 2010, p. 565-578; Natan Sznaider, "Rewriting the Persian Letters ", British Journal of Sociology, vol. 61, n 3, 2010, p. 627-633; Joy Yueyue Zhang, "The Cosmopolitization of Science: Experience from Chinese Stem Cell Scientists ", Soziale Welt, vol. $61, \mathrm{n}^{\text {os }} 3-4,2010$, p. 257-276. 
différentes régions ou pour différentes ethnies ou religions - sont exposés à ces nouvelles forces civilisationnelles en des modes mutuellement exclusifs. Que ce soit voulu ou non, ce sont là les préoccupations de chaque nation, de chaque personne parce qu'elles sont structurellement imbriquées dans le nouveau processus de civilisation que je nomme " cosmopolitisation (réfléchie) »; et la condition civilisationnelle qui est ainsi partagée à travers le globe est une "modernité (réflexive ou) seconde ${ }^{10}$ ".

Les efforts isolationnistes - qu'ils s'expriment en termes de commerce protectionniste, de fondamentalisme religieux, de fondamentalisme national, de contrôle d'Internet et des médias, ou autrement - sont aisément sujets à des condamnations morales internationales (et sont, dans une certaine mesure, inefficaces). En fait, il ne relève pas de choix politiques volontaires d'accepter ou de refuser ces forces puisqu'il s'agit de réflexes mondiaux (comme le démontre le conflit des caricatures) - c'està-dire qui surviennent compulsivement à travers l'impératif cosmopolite : coopère ou échoue!

La cosmopolitisation peut être étudiée dans plusieurs champs : en relation avec la migration, la classe, la famille, la science, l'État, l'individu, la région, les religions, les relations internationales; mais elle l'est aussi de façon très évidente quand il s'agit de l'inégalité sociale et des risques mondiaux.

\section{Reconfigurer l'inégalité sociale à l'ère des changements climatiques}

La perspective nationale sur l'inégalité est orientée vers l'intérieur. Elle s'arrête aux frontières de l'État-nation. Les inégalités sociales peuvent fleurir et prospérer de l'autre côté de la clôture du jardin

10 Ulrich Beck, The Cosmopolitan Vision, Cambridge (UK) / Malden (MA), Polity Press, 2006; Ulrich Beck, Anthony Giddens et Scott Lash, Reflexive Modernization: Politics, Tradition and Aesthetics in the Modern Social Order, Cambridge (UK), Polity Press, 1994; Ulrich Beck et Edgar Grande, «Varieties of Second Modernity: The "Cosmopolitan Turn" in Social and Political Theory and Research ", op. cit.; Chang, Kyung-Sup, "The Second Modern Condition? Compressed Modernity as Internalized Reflexive Cosmopolitanism ", op. cit. 
national; ce sera, au mieux, une cause de scandale moral, mais ce sera politiquement hors de propos. On doit faire une distinction claire, donc, entre la réalité de l'inégalité sociale et le problème politique de l'inégalité sociale ${ }^{11}$. Les frontières nationales distinguent nettement entre une inégalité politiquement pertinente et une autre qui est déplacée. Les inégalités au sein des sociétés nationales donnent lieu à des perceptions étirées; en même temps, les inégalités entre les sociétés nationales sont atténuées. La "légitimation » des inégalités mondiales repose sur un « regarder d'une autre manière » institutionnalisé. Le regard national est "dispensé " ( freed ") de se pencher sur la misère du monde. Il opère par une double exclusion : il exclut les exclus. Et les sciences sociales de l'inégalité, lesquelles assimilent l'inégalité à l'inégalité dans l'État-nation, sont complices. Il est étonnant, en effet, de constater à quel point les inégalités mondiales sont "légitimées " sur la base d'une entente tacite entre le gouvernement d'un État-nation et la sociologie d'un État-nation une sociologie programmée pour travailler sur une base d'État-nation et prétendant ne pas faire intervenir de valeurs.

Mon argument est le suivant : le principe de performance rend légitime l'inégalité nationale, le principe d'État-nation rend légitime l'inégalité mondiale (sous une autre forme). Comment? Les inégalités entre pays, régions et États sont considérées comme étant politiquement incomparables. D'un point de vue délimité par un État-nation, les comparaisons politiquement pertinentes peuvent être faites seulement à l'intérieur d'un État-nation, jamais entre États. De telles comparaisons, qui rendent l'inégalité politiquement explosive, présupposent des normes nationales d'égalité. C'est la raison pour laquelle même des différences massives de revenu entre personnes avec les mêmes qualifications mais ayant différentes nationalités n'ont de force politique que si elles peuvent être reliées a un horizon de perception d'égalité commune. Un tel cadre commun n'apparaît que si les personnes

11 Ulrich Beck, « Remapping Social Inequalities in an Age of Climate Change: for a Cosmopolitan Renewal of Sociology ", Global Networks, vol. 10, n 2, 2010, p. 165-181. 
appartiennent à la même nation, ou à l'Union européenne, ou sont employées dans la même compagnie, même s'il s'agit de différentes filiales nationales.

Pourtant, c'est précisément ce que le regard national atténue : plus les normes d'égalité s'étendent dans le monde, plus l'inégalité mondiale est dépouillée des fondements légitimes qui permettent institutionnellement de regarder au loin. Les riches démocraties portent la bannière des droits humains dans les coins les plus éloignés de la terre, sans prendre en considération que la défense des frontières nationales, avec lesquelles elles veulent repousser les courants de migrants, perd ainsi de sa légitimité. Bon nombre de migrants prennent au sérieux le droit humain proclamé d'égalité de la mobilité et croisent des pays et des États - dont les inégalités internes sont souvent graves - qui veulent que les normes d'égalité s'arrêtent brusquement à leurs frontières fortifiées. En d'autres mots, cela signifie que la conception d'inégalité sociale, fondée sur des principes de nationalité et d'étatisme (statehood), oriente l'analyse de manière inadéquate. La plupart des théoriciens des classes, y compris Pierre Bourdieu, qui a réfléchi de façon considérable sur la mondialisation durant ses dernières années, identifient société de classes à État-nation. Il en va de même d'Immanuel Wallerstein ${ }^{12}$ et de John $\mathrm{H}$. Goldthorpe $^{13}$, en fait aussi de presque tous les sociologues qui s'intéressent à l'inégalité sans recourir à la théorie des classes (et incidemment aussi de ma thèse sur l'individualisation ${ }^{14}$ ).

Sans le concept de vulnérabilité sociale " locationale ", il est impossible de comprendre les implications du changement

12 Immanuel Wallerstein : The Modern World-System, Volume I, Capitalist Agriculture and the Origins of the European World-Economy in the Sixteenth Century, New York/Londres, Academic Press, 1974; The Modern WorldSystem, Volume II, Mercantilism and the Consolidation of the European World-Economy, 1600-1750, New York, Academic Press, 1980; The Modern World-System, Volume III, The Second Great Expansion of the Capitalist WorldEconomy, 1730-1840s, San Diego, Academic Press, 1989.

13 John H. Goldthorpe, "Globalisation and Social Class ", West European Politics, vol. 25, n 3, 2002, p. 1-28.

14 Beck, Ulrich, Risk Society: Towards a New Modernity, London / Newbury Park / New Delhi, Sage Publications, 1992. 
climatique sur la reconfiguration cosmopolite des inégalités. Au cours des dernières années, toutefois, la vulnérabilité sociale est devenue une dimension clé de l'analyse sociologique des inégalités dans une société liée au fait du risque mondial : la mondialisation du capitalisme industriel produit une exposition inégale radicale à des risques à peine définissables, et les inégalités qui en résultent doivent largement être comprises comme une expression et un produit des relations de pouvoir dans un contexte national et mondial. La vulnérabilité sociale est un concept synthétique, englobant des moyens et des possibilités que les individus, les communautés ou des populations entières ont à leur disposition afin de faire face - ou non - aux menaces de changement climatique (ou de crises financières).

Comprendre la vulnérabilité, c’est certainement entretenir une relation cruciale avec l'avenir, mais c'est aussi saisir l'histoire en profondeur. Les " blessures culturelles ", qui, par exemple, sont les conséquences d'un passé colonial, constituent une partie importante de l'arrière-fond à partir duquel on comprend les conflits liés au caractère transfrontalier du changement climatique $^{15}$. On peut en trouver les effets au sommet de Copenhague sur le climat qui a eu lieu en décembre 2009. Les pays en voie de développement - comme le Brésil, l'Afrique du Sud et l'Inde (selon les vœux des États-Unis, tous les partenaires d'une éventuelle alliance mondiale des démocraties pour faire obstacle aux pouvoirs autoritaires) - ont décidé que leur statut en tant que nations postcoloniales était plus important que leur statut de démocraties. Comme la Chine, ils soutiennent qu'il est fondamentalement injuste de plafonner les émissions de gaz à effet de serre des pays pauvres à un niveau plus bas que celui des émission des États-Unis ou de l'Union européenne; d'autant que l'Occident industrialisé est largement responsable des conséquences des risques climatiques dont ils souffrent. De façon révélatrice, les leaders aussi bien brésiliens que chinois ont fait la même blague incisive - comparant les États-Unis à un homme riche qui, après

15 Jeanne X. Kasperson et Roger F. Kasperson, The Social Contours of Risk, 2 volumes, Londres / Sterling (VA), Earthscan, 2005. 
s'être goinfré à un banquet, invite ses voisins à prendre un café et leur demande de partager la facture! Ainsi, à cause de la question de (in)justice qui est incorporée à la cosmopolitique du changement climatique, il n'y a pas de New Deal vert mondial jusqu’à présent et les États-Unis sont en train de perdre le monde libre.

La reconfiguration cosmopolite de l'inégalité doit prendre en compte trois paramètres :

i. la mondialisation de l'inégalité sociale ne peut plus être détachée de la mondialisation de l'égalité sociale;

ii. les sciences sociales de l'inégalité sociale ne peuvent plus dépendre de la prémisse d'une distinction entre national et international; l'équation de l'inégalité sociale et nationale que le nationalisme méthodologique adopte est devenue une source d'erreur par excellence;

iii. la prémisse de base des sciences sociales, la distinction entre inégalité sociale et inégalité naturelle, est devenue indéfendable.

Quiconque pense ces trois composants ensemble rencontre un paradoxe : plus sont reconnues mondialement les normes d'égalité, plus deviennent insolubles le problème climatique et les inégalités socio-écologiques. Ce n'est pas là une perspective joyeuse! Mais c'est précisément ce réalisme incorruptible, ouvert sur le monde, qui est désigné par le concept de "perspective cosmopolite " (la perspective de l'acteur) et par le cosmopolitisme méthodologique (la perspective de l'observateur). On n'a pas ici affaire à une rhétorique officielle de fraternité mondiale, mais à des perceptions aiguisées dans la vie quotidienne, dans la politique et dans le savoir qui sont destinées aux forces explosives sans bornes de l'inégalité sociale à l'ère du changement climatique et de la mondialisation. Ce réalisme cosmopolite envisage la possibilité que les gouvernements des États-nations s'affirment militairement ou même deviennent fous furieux devant la perte d'autonomie qu'ils connaîtraient. 
En résumé, le nationalisme méthodologique repose sur une double présupposition de coïncidence : d'un côté, la coïncidence des frontières territoriales, politiques, économiques, sociales et culturelles; d'un autre, la coïncidence de la perspective de l'acteur et de celle du spécialiste des sciences sociales. La prémisse du nationalisme normatif-politique des acteurs devient sans en être le reflet la prémisse de la perspective de l'observateur des sciences sociales. Ces présuppositions de coïncidence se renforcent mutuellement. La tendance historique, toutefois, va dans une direction opposée : les frontières territoriales, étatiques, économiques, sociales continuent d'exister; mais elles ne sont plus adjacentes! Le nécessaire changement paradigmatique peut se construire sur trois idées :

- les classes sociales sont seulement une des formes historiques de l'inégalité;

- l'État-nation est seulement un des cadres historiques d'interprétration;

- "la fin de la société nationale de classes " ne signifie pas " la fin de l'inégalité sociale ", au contraire : l'inégalité est exacerbée dans les sphères aussi bien nationale que transnationale.

Aujourd'hui, on assiste à une sorte de répétition du processus que Max Weber a observé quand il a analysé les origines du capitalisme moderne ${ }^{16}$. Sauf que, maintenant, ce processus a cours à une échelle mondiale. Weber a dit que nous devions porter notre attention sur la séparation entre le foyer familial et le capitalisme d'entreprise - c'est-à-dire sur l'émancipation des intérêts économiques. À présent, nous témoignons de l'émancipation des intérêts économiques par rapport aux liens nationaux et aux organismes de contrôle. Cela signifie la séparation $d u$ pouvoir et de la politique. L'État-nation émergent a développé des institutions de pouvoir et de politique qui étaient capables de limiter les blessures du capitalisme industriel moderne. Cela a été

16 Max Weber, "Die protestantische Ethik und der Geist des Kapitalismus ", dans Gesammelte Aufsätze zur Religionssoziologie, vol. 1, Tübingen, J. C. B. Mohr, 1988. 
possible à l'intérieur des frontières territoriales de l'État-nation et a constitué une sorte de mariage entre le pouvoir et la politique, un mariage qui se termine maintenant en divorce. En conséquence, le pouvoir devient plus diffus, en partie transféré dans le cyberespace, aux marchés financiers et au capital mobile, en partie déplacé vers les individus qui ont maintenant à faire face seuls aux risques concomitants. Et à présent, il n'y a pas d'équivalent de la vision d'un État-nation souverain.

\section{Le moment cosmopolite de la société du risque}

La théorie de la société du risque mondial soutient que les sociétés modernes sont modelées par de nouvelles sortes de risques, qu'elles fabriquent des incertitudes, que leurs fondements sont ébranlés par l'anticipation mondialisée de catastrophes mondiales. De telles perceptions de risque mondial ont trois caractéristiques $^{17}$ :

i. la dé-localisation : ses causes et ses conséquences ne sont pas limitées à un lieu ou à un espace géographiques, elles sont en principe omniprésentes;

ii. l'incalculabilité : ses conséquences sont en principe incalculables; au fond, il s'agit d'une question de risques " hypothétiques", risques, ce qui n'est pas le moindre problème, qui ont pour fondement la science, qui sont causés par le non-su et la dissension normative;

iii. la non-dédommageabilité : le rêve de sécurité de la première modernité reposait sur la maîtrise des conséquences malsaines et des dangers liés aux décisions; les accidents pouvaient survenir pour autant qu'ils étaient considérés comme dédommageables et parce qu'ils apparaissaient tels. À partir du moment où le système de finance mondial s'est effondré, où le climat a irrévocablement changé, où les groupes terroristes

17 Ulrich Beck, World at Risk, Cambridge (UK) / Malden (MA), Polity Press, 2009. 
disposent des armes de destruction de masse, alors il est trop tard. Compte tenu de cette nouvelle qualité des "menaces pour l'humanité " - soutient François Ewald $^{18}$-, la logique du dédommagement se brise et elle est remplacée par le principe de précaution par la prévention. Non seulement donne-t-on alors préséance à la prévention sur le dédommagement, mais aussi on essaie d'anticiper et de prévenir les risques dont l'existence n'a pas été démontrée.

Qu'entend-on alors par «moment cosmopolite » de la société du risque? Le changement climatique exacerbe les inégalités entre les pauvres et les riches, entre le centre et la périphérie - mais les dissout simultanément. Plus est grande la menace planétaire, moins il devient possible pour les mieux nantis et pour les plus forts de l'éviter. Le changement climatique est à la fois hiérarchique et démocratique. Le changement climatique est d'une pure ambivalence, il impose l'impératif cosmopolite : coopère ou échoue.

Le réalisme courant, d'une séduisante naïveté catastrophiste, se trompe. Les risques climatiques ne sont pas identiques aux catastrophes climatiques ${ }^{19}$. Les risques climatiques sont l'anticipation au présent des catastrophes futures. $\mathrm{Ce}$ " futur présent " des risques climatiques est réel; le "futur futur " des catastrophes climatiques, par contre, est (encore) irréel. Pourtant, même l'anticipation du changement climatique aménage une transformation fondamentale dans le ici et maintenant. Depuis qu'on a cessé de se demander si le continuel changement climatique était produit par l'humain et s'il avait des effets catastrophiques sur la nature et la société, les cartes dans la société et dans la politique ont été redistribuées à l'échelle du monde. C'est pourquoi le changement climatique conduit, à n'en pas douter, directement et inévitablement vers l'apocalypse, en même temps qu'il fournit

18 François Ewald, "The Return of Descartes' Malicious Demon: An Outline of a Philosophy of Precaution ", dans Tom Baker et Jonathan Simon (dir.), Embracing Risk: The Changing Culture of Insurance and Responsibility, Chicago/Londres, University of Chicago Press, 2002, p. 273-301.

19 Ulrich Beck, World at Risk, op. cit. 
l'occasion de surmonter l'étroitesse nationale de la politique et de développer une realpolitik dans l'intérêt national.

Dans la mesure où un public mondial devient conscient du fait que l'État-nation est miné par les risques mondiaux (changements climatiques, crises économiques) qui relient les nations sous-développées et développées les unes aux autres, alors quelque chose d'historiquement nouveau peut émerger, à savoir une vision cosmopolite dans laquelle les personnes se voient ellesmêmes comme faisant partie à la fois d'un monde menacé, d'une part, et de leur histoire locale et de leur situation de survie, d'autre part.

Ainsi, le changement climatique - comme l'ancien cosmopolitisme (le stoïcisme), comme le ius cosmopolitica des Lumières (Emmanuel Kant) ou les crimes contre l'humanité (Hannah Arendt, Karl Jaspers) - donne lieu à un moment et à un momentum cosmopolites. Les risques mondiaux impliquent qu'on soit confronté à l'autre global. Ils abolissent les frontières nationales et mélangent le local avec l'étranger, non pas comme conséquence d'une migration, mais plutôt comme conséquence d'une interconnectivité et des risques. Tous les jours, la vie devient cosmopolite : les personnes doivent mener et comprendre leur vie dans un échange avec les autres, et non plus de façon exclusive dans une interaction avec leurs semblables.

Afin de comprendre et de conceptualiser le phénomène, on doit abandonner le présupposé selon lequel la construction des communautés n'est possible que sur fond d'une intégration positive via des valeurs et des normes communes, et on doit s'accoutumer à l'idée que la construction des communautés peut aussi résulter de conflits entre des valeurs négatives (crises, risques, menaces d'anéantissement). Ainsi, on doit distinguer entre les risques qu'on provoque soi-même et ceux que provoque l'étranger. Dans les cas où on se met soi-même en péril, les personnes qui décident des risques et celles qui sont affectées par ces risques appartiennent au même groupe social et culturel; c'est vrai pour les trajectoires européenne et étatsuniennes de la modernisation. À l'opposé, dans le cas où on est mis en danger 
par l'étranger, les décideurs et les personnes qui sont affectées appartiennent à différents pays, à différentes cultures, à différentes régions du monde; cela correspond à la trajectoire postcoloniale de la modernisation.

Ainsi la question de l'inégalité et de la règle se situe dans la logique du risque mondial lui-même. Le risque requiert une décision, donc un décideur, et génère une asymétrie radicale entre les personnes qui prennent le risque, le définissent et en profitent et celles qui doivent accepter le risque et subir les " effets secondaires invisibles " des décisions qui ont été prises par d'autres, peut-être même en payant de leur vie, n'ayant pas eu la chance de participer au processus de décision. Il est, en effet, d'une grande pertinence cosmopolitique de distinguer la mise en péril dont on est soi-même le responsable et celle qui est provoquée par l'étranger puisque les relations entre les régions du monde entier sont dépeintes comme des relations de pouvoirs caractérisées par l'externalisation des menaces contre les autres qu'on produit soi-même. Le riche et puissant produit les risques associés au changement climatique et en bénéficie alors que le pauvre et faible souffre de leur existence, des effets secondaires de nos décisions.

\section{Le cosmopolitisme méthodologique : le problème de l'unité de recherche}

Le tournant cosmopolite révèle la question clé suivante : quelle unité de recherche constitue la base (appropriée) de la théorie et de la recherche sociales? Ou, plus précisément : comment les unités de recherche, au-delà du nationalisme méthodologique, peuvent-elles être découvertes et définies, nous permettre de comprendre les processus de cosmopolitisation et de faire des analyses comparées des variations dans la modernité cosmopolite? Quels sont les points de référence des analyses des sciences sociales si, d'un côté, on veut la libérer du "conteneur " de l'État-nation mais, d'un autre côté, ne pas se réfugier dans les concepts abstraits de la "société mondiale "? La recherche empirique dans des disciplines aussi différentes que l'anthropologie, 
la géographie ou les sciences politiques a, au cours des dernières années, développé un grand nombre de concepts, tous dans le but de fracturer l'équation "société/nation/État ", supposément " naturelle ». La conceptualisation de l'Atlantique noir de Paul Gilroy $^{20}$, l'identification de la cité mondiale de Saskia Sassen's ${ }^{21}$, la notion de "scapes " d'Arjun Appadurai ${ }^{22}$, le concept d'âge mondial de Martin Albrow ${ }^{23}$ et notre propre analyse d'une Europe cosmopolite ${ }^{24}$ sont des exemples d'une telle recherche.

Cette variété de concepts et d'approches peut être utilement systématisée en procédant à deux distinctions. D'un côté, on peut distinguer de nouvelles unités de recherche selon qu'elles se réfèrent aux processus de transnationalisation ou aux structures transnationales. À titre d'exemple de la première référence, on peut songer aux processus de migration transnational; comme exemple de la seconde, on peut évoquer les " communautés de diaspora " dans lesquelles les migrants créent de nouvelles formes de (co-)existence transnationale. Une seconde distinction a trait à l'étendue et au lieu de la cosmopolitisation. L'étendue implique la question du rôle que l'unité de recherche du national, c'est-àdire de l'État-nation, avec les cultures de la nation, etc., joue (encore) à l'ère de la cosmopolitisation. Le lieu implique la question du niveau auquel l'accent est mis sur la cosmopolitisation - par delà le national : les régions du monde, les religions du monde; en-dessous du niveau du national : le local, la famille, l'individu, l'entreprise, le travail, etc. Il est possible, bien sûr, que l'État-nation et le national continuent d'exister, mais perdent leur position de monopole épistémologique, par exemple, parce qu'ils deviennent intégrés dans de nouvelles formes d'organisation

$20 \quad$ Paul Gilroy, Black Atlantic: Modernity and Double Consciousness, Cambridge (MA), Harvard University Press, 1993.

21 Saskia Sassen, The Global City: New York, London, Tokyo, Princeton, Princeton University Press, 1991.

22 Arjun Appadurai, Modernity at Large: Cultural Dimensions of Globalization, Minneapolis, University of Minnesota Press, 1996.

23 Martin Albrow, The Global Age: State and Society Beyond Modernity, Cambridge (UK) / Stanford (CA), Polity Press / Stanford University Press, 1996.

24 Ulrich Beck et Edgar Grande, Cosmopolitan Europe, op. cit. 
politique et d'ordre social. Les conséquences méthodologiques seraient qu'on doive trouver de nouvelles unités de recherche qui contiennent le national, mais qui ne coïncident plus avec le national. On décrirait cela comme un « encastrement du national ».

Le remplacement de l'objet de recherche du national par d'autres points focaux représente une possibilité plus radicale; cette transformation ne signifie pas que le national devienne obsolète ou hors de propos ("que le national soit remplacé »).

On suppose ou soutient souvent que l'importance nouvellement ou continûment grandissante de l'État légitime le nationalisme méthodologique. Mais cette position repose sur une équation fausse de la perspective de l'acteur et de celle de l'observateur. Même si l'État-nation gagne au plan fonctionnel en importance, il peut perdre sa position de monopole épistémologique en tant qu'unité de recherche puisque les recherches sur le processus de cosmopolitisation ne peuvent être menées à bien que si d'autres points focaux se déplacent sur le devant de la scène. De cette façon, on peut construire un tableau pour présenter la boîte à outils conceptuelle de cosmopolitisme méthodologique. Qu'on me permette de l'illustrer brièvement : 


\section{Tableau :}

Unités d'analyse du cosmopolitisme méthodologique

\begin{tabular}{|c|c|c|}
\hline & Processus & Structures \\
\hline $\begin{array}{c}\text { Encastrer le } \\
\text { national }\end{array}$ & $\begin{array}{l}\text { "Modernisation } \\
\text { comprimée }^{25} " ; \text { " europé } \\
\text { cosmopolitisation anisation } \\
\text { cosmopolite }^{26} » ; \\
\text { " cosmopolitisation } \\
\text { recursive }^{27} "\end{array}$ & $\begin{array}{l}\text { Régimes politiques } \\
\text { transnationaux }{ }^{28} ; \\
\text { assemblages mondiaux }{ }^{29} ; \\
\text { Régions du monde }^{30}\end{array}$ \\
\hline $\begin{array}{c}\text { Remplacer le } \\
\text { national }\end{array}$ & $\begin{array}{l}\text { "navires en mouvement }{ }^{31} » ; \\
\text { "chaînes } \\
\text { d'approvisionnement du } \\
\text { capitalisme }{ }^{32} " \text {; " innovations } \\
\text { cosmopolites }^{33} "\end{array}$ & $\begin{array}{l}\text { Religions du monde; } \\
\text { réseaux de migrants } \\
\text { transnationaux }{ }^{34} \text {; zones } \\
\text { frontalières; cité mondiale; } \\
\text { le local; la famille; } \\
\text { l'individuel }\end{array}$ \\
\hline
\end{tabular}

25 Kyung-Sup Chang, "Compressed Modernity and Its Discontents: South Korean Society in Transition ", Economy and Society, vol. 28, n' 1, 1999, p. 30-55; Chang, Kyung-Sup, «The Second Modern Condition? Compressed Modernity as Internalized Reflexive Cosmopolitanism ", op. cit. Ulrich Beck et Edgar Grande, Cosmopolitan Europe, op. cit.

27 Daniel Levy, "Recursive Cosmopolitization: Argentina and the Global Human Rights Regime ", op. cit.

28 Edgar Grande, "Vom Nationalstaat zum transnationalen Politikregime Staatliche Steuerungsfähigkeit im Zeitalter der Globalisierung ", op. cit.

29 Saskia Sassen, Territory, Authority, Rights: From Medieval to Global Assemblages, op. cit.

30 Peter J. Katzenstein, A World of Regions: Asia and Europe in the American Imperium, Ithaca, Cornell University Press, 2005.

31 Paul Gilroy, Black Atlantic: Modernity and Double Consciousness, op. cit.

32 Anna Tsing, "Supply Chains and the Human Condition ", Rethinking Marxism, vol. 21, nº 2, 2009, p. 148-76.

33 David Tyfield et John Urry, « Cosmopolitan China? Lessons from International Collaboration in Low-Carbon Innovation ", British Journal of Sociology, vol. 60, no 4, 2009, p. 793-812.

34 Nina Glick Schiller, "A Global Perspective on Migration and Development », Social Analysis, vol. 53, n 3, 2009, p. 14-37. 
a) Navires en mouvement : remplacer le national par les processus transcontinentaux

Gilroy est l'auteur d'un des premiers et des plus radicaux dépassements du nationalisme méthodologique (bien que son travail n'ait pas été produit dans cet esprit). Dans son étude déterminante, il n'a pas adopté le " conteneur » national; il a plutôt pris un espace transcontinental, l'Atlantique noir ${ }^{35}$, comme champ pour sa théorisation de recherche empirique. Gilroy a soutenu de façon convaincante que les idées de nation, de nationalité et de biens nationaux n’ont pas de conséquences épistémologiques : elles confirment l'existence d'un programme de recherche et d'une pratique animés par un « intérieurisme culturel » fondé sur une différence ethnique essentialiste. Le point de départ de Gilroy n'est pas une entité fixe; au contraire, l'image d'un navire en mouvement entre l'Europe, l'Amérique, l'Afrique et les Caraïbes se veut le principe symbolique central d'organisation de sa recherche.

Dans sa propre recherche sur les formes des chaînes d'approvisionnement transnationales du capitalisme, Anna Tsing a adopté une approche similaire qui remplaçait le national comme unité de base par des processus transnationaux. Contrairement aux théories sur la convergence et l'homogénéité croissante du capitalisme, son analyse souligne le rôle fondamental de la différence dans la mobilisation du capital, du travail et d'autres ressources. Le centre et l'unité d'analyse dans sa recherche à nouveau n'est pas une entité fixe, c'est un processus : la mobilisation du travail dans les chaînes d'approvisionnement, puisqu'elle dépend de la performance du genre, de l'ethnie, de la nationalité, de la religion et du statut de citoyenneté. Son analyse révèle que la diversité est d'une importance centrale pour le capitalisme mondial et qu'elle n'est pas simplement décorative.

35 Paul Gilroy, Black Atlantic: Modernity and Double Consciousness, op. cit. 
b) Les réseaux transnationaux et les zones frontalières : remplacer le national par de nouvelles structures nationales

Au cours de la dernière décennie, il y a eu une quantité substantielle de recherches dans le domaine des études transnationales ${ }^{36}$. Sans tenir compte de divers objets de recherche et de disciplines universitaires, elles ont en commun de remplacer le national en tant qu'unité d'analyse par de nouvelles structures qu'on peut observer à une multiplicité de niveaux. La plus innovatrice est celle qui porte sur la migration transnationale et qui est menée par Schiller ${ }^{37}$ et d'autres chercheurs ${ }^{38}$. Ces recherches privilégient une perspective constructiviste des espaces territoriaux dans laquelle les formations sociales transnationales - comme les réseaux transnationaux, les groupes de parenté, les organisations migrantes et les communautés de diaspora - franchissent les territoires des États- nations et les chevauchent, sans nécessairement avoir une portée mondiale.

Dans les études historiques, on a récemment introduit une approche similaire : l'historiographie transnationale. Cette approche met en question la concentration sur l'État-nation, sur l'histoire des États, des zones frontalières et des flux de transferts interrégionaux. Elle se penche plutôt sur la configuration et sur les fonctions politiques des zones frontalières. Elle permet de faire une distinction analytique entre les processus de formation des États et la territorialisation. Contrairement à ce qui est fait dans une perspective centrée sur l'État-nation, elle suggère d'analyser les frontières de l'État-nation non pas comme s'il s'agissait de limites claires, mais comme des aires frontalières mouvantes et inclusives $^{39}$. Bien sûr, il y a eu, dans les études historiques, depuis

36 Sanjeev Khagram et Peggy Levitt, Peggy (dir.), The Transnational Studies Reader: Intersections and Innovations, Londres/New York, Routledge, 2008; Ludger Pries (dir.), Rethinking Transnationalism: The Meso-Link of Organizations, Abingdon (Oxon) / New York (NY), Routledge, 2008; Steven Vertovec (dir.), Transnationalism, Londres, Routledge, 2009.

37 Nina Glick Schiller, "A Global Perspective on Migration and Development ", op. cit.

38 Voir Steven Vertovec (dir.), Transnationalism, op. cit.

39 Anthony G. Hopkins (dir.), Globalization in World History, New York, W. 
le XIX ${ }^{\mathrm{e}}$ siècle, une tension entre les travaux centrés sur le national et les études thématiques sur, par exemple, le féodalisme, les Lumières, la révolution industrielle, le fascisme; il y a eu l'étude de la mouvance des régions frontalières qui est assez vieille elle aussi, on en a un exemple dans le Danube de Claudio Magris ${ }^{40}$ - quoiqu'il s'agisse d'un ouvrage guidé davantage par la littérature que par les sciences sociales -; et il y a eu, et ce n'est pas le moindre, le travail innovateur de Karl Schögel ${ }^{41}$. Pour le dire autrement, l'historiographie transnationale fait bonne impression; mais elle représente surtout une réponse à une question américaine, elle a pour fin d'épuiser un provincialisme spécifique étatsunien.

c) Encastrer le national dans les structures et les processus transnationaux nouveaux

Les "régimes politiques transnationaux" en sont un premier exemple ${ }^{42}$. Les travaux renvoient aux nouvelles formes de construction d'institutions transnationales qui se sont établies elles-mêmes dans un certain nombre de sphères de réglementation mondiale comme la protection du climat, la réglementation d'Internet ou l'imposition des entreprises transnationales. Ces

W. Norton, 2002; David Thelen, "The Nation and beyond: Transnational Perspectives on United States History ", Journal of American History, vol. 86, $\mathrm{n}^{\circ}$ 3, 1999, p. 965-75; Ian Tyrrell, " Making Nations / Making States: American Historians in the Context of Empire ", Journal of American History, vol. 86, n 3, 1999, p. 1015-1044.

40 Claudio Magris, Danube: A Sentimental Journey from the Source to the Black Sea, Londres, Harvill Press, 1999.

41 Karl Schlögel, Im Raume lesen wir die Zeit: Über Zivilisationsgeschichte und Geopolitik, München, Carl Hanser Verlag, 2003.

42 Edgar Grande, "Vom Nationalstaat zum transnationalen Politikregime Staatliche Steuerungsfähigkeit im Zeitalter der Globalisierung ", dans Ulrich Beck et Christoph Lau (dir.), Entgrenzung und Entscheidung. Was ist neu an der Theorie reflexiver Modernisierung?, Frankfurt am Main, Suhrkamp, 2004, p. 384-401; Edgar Grande et Louis W. Pauly (dir.), Complex Sovereignty: Reconstituting Political Authority in the Twenty-first Century, Toronto / Buffalo / Londres, University of Toronto Press, 2005; Edgar Grande, Markus König, Patrick Pfister et Paul Sterzel, « Globalization, Political Transnationalization, and the Future of the Nation-State - A Comparison of Transnational Policy Regimes ", dans S.A. Schirm (dir.), Globalization: State of the Art and Perspectives, Londres/New York, Routledge, 2007, p. 98-121. 
institutions représentent des relations d'interaction qui ne sont pas définies par un espace national d'opération mais qui le sont plutôt par des problèmes de réglementation pratiques; elles intègrent des groupes participants très différents et extrêmement variables et s'étendent à divers niveaux territoriaux. En conséquence, ce sont ces régimes politiques qui deviennent les unités de recherche pertinentes pour une analyse empirique des politiques transnationales. Ce qui est crucial, ici, il est vrai, c'est que ces nouvelles institutions ne remplacent pas les États-nations; elles les intègrent. Les États-nations sont encastrés dans les nouveaux systèmes transnationaux de la réglementation, et l'une des tâches les plus importantes de l'analyse empirique devient d'examiner l'importance spécifique des États-nations dans ces institutions. Là où l'État-nation continue de dominer, comme on peut maintenant l'observer dans le cas des réglementations climatiques, le transnational menace de devenir la « vitrine du national ".

On trouve un second exemple des raisons pour lesquelles il peut être nécessaire de continuer à choisir l'État-nation comme unité de recherche et de dépasser néanmoins l'étroitesse du nationalisme méthodologique dans les travaux de Daniel Levy ${ }^{43}$, de Natan Sznaider ${ }^{44}$, de Levy et Sznaider ${ }^{45}$ et dans ceux de Saskia Sassen $^{46}$. Ces travaux montrent que les processus de mondialisation dérivent des transformations au sein des États-nations et trouvent là leur dynamique, qu'il s'agisse de la croissance du marché mondial du capital ou des réseaux de militants des droits humains $s^{47}$. Bref, l'espace national devient une "vitrine du mondial » hautement complexe. Précisément à cause de l'interconnectivité du monde, plusieurs choses commencent locale-

43 Daniel Levy, "Recursive Cosmopolitization: Argentina and the Global Human Rights Regime ", op. cit.

44 Natan Sznaider, "Rewriting the Persian Letters ", op. cit.

45 Daniel Levy et Natan Sznaider, Human Rights and Memory, University Park (PA), Penn State University Press, 2010.

46 Saskia Sassen, Territory, Authority, Rights: From Medieval to Global Assemblages, Princeton, Princeton University Press, 2006.

47 Fuyuki Kurasawa, The Work of Global Justice: Human Rights as Practices, Cambridge, Cambridge University Press, 2007. 
ment, même si, à l'occasion, cela se fait dans une proximité non localisée. Ainsi ce n'est plus national ni mondial, mais bien cosmopolite.

\section{Perspective : le changement de paradigme dans les sciences sociales}

Il est évident que le cadre de référence de l'État-nation, qui est pris pour acquis - "nationalisme méthodologique »-, empêche les sciences sociales de comprendre et d'analyser les dynamiques, les ambivalences et les chances de la condition humaine cosmopolite dans le XXI ${ }^{e}$ siècle. Un nationaliste méthodologique, aussi bien qu'un politicien national, qui tentent de gérer les risques mondiaux dans l'isolement ressemblent à un ivrogne qui, durant une nuit noire, essaie de trouver le porte-monnaie qu'il a perdu sous la lumière d'un réverbère. À la question " avez-vous perdu votre porte-monnaie ici ", il répond «non, mais sous la lumière du réverbère, je peux au moins le chercher ».

Mais la sociologie et la science politique ne sont pas les seules disciplines sous pression, l'économie l'est aussi. On a affaire à une situation de vacance : on est à la recherche d'un théoricien qui serait un monsieur Keynes, mais au plan transnational. On a maintenant besoin d'une réglementation réfléchie et intelligente (philosophique) à une échelle mondiale, incluant de nouveaux acteurs comme les entreprises transnationales, la Banque mondiale, le Fonds monétaire international, Attac, Greenpeace, etc. Les États-Unis ont un besoin équivalent pour des institutions politiques beaucoup plus fortes - un ministre européen des affaires économiques - qui serait un complément à la banque centrale. Mais ce dont on a le plus grand besoin, c'est d'une nouvelle théorie de l'économie mixte, formulée pour le marché mondial d'aujourd'hui, puisque le défunt système keynésien a été fabriqué pour les économies nationales d'après-guerre.

On est sous le charme de la sociologie, dont les fondements ont été conçus et développés au cours des cent dernières années. Le premier siècle de la sociologie est terminé. En route vers le deuxième, qui a maintenant débuté; l'espace de l'imagination et 
de la recherche sociologiques doit être désenclavé et défini à nouveau, c'est-à-dire qu'il doit être désenclavé pour accueillir la constellation cosmopolite. Apprendre sur les autres n'est pas simplement un acte d'ouverture d'esprit; c'est une partie intégrante de l'apprentissage et de la compréhension de la réalité de soi-même ou même un facteur essentiel pour arriver à se voir soi-même comme autre. Une sociologie cosmopolite est une sociologie qui se reflète dans des prémisses ontologisées, dans des dualismes de la sociologie de l'État-nation - comme national et international, nous et eux, interne et externe -; cette sociologie se dote d'une grammaire significative pour la politique et le social, elle recompose la recherche de telle façon qu'elle acquière un nouveau cadre de référence pour toutes sortes de sujets. Une sociologie cosmopolite se distingue clairement d'une sociologie universaliste parce qu'elle ne débute pas avec quelque chose d'abstrait (qui dérive normalement d'une expérience et d'un contexte historiques européens, par exemple "société » ou " société mondiale " ou "système mondial » ou " autonomie individuelle ", etc.). Ce sont plutôt des concepts clés comme dépendance, interdépendance, interconnectivité qui figurent sur le devant de la scène, ainsi que les questions méthodologiques qu'ils posent : comment peut-on faire de la recherche sur un monde interdépendant? La sociologie cosmopolite, donc, ouvre d'indispensables nouvelles perspectives sur des contextes apparemment isolés, familiers, locaux et nationaux. Mais elle ne fait que débuter; il y a plus de problèmes que de solutions, plus de questions que de réponses. Mais nous, les sociologues, ne sommes pas les premiers dans cette quête. Avec cette nouvelle "vision cosmopolite », nous suivons les trajectoires empiriques et méthodologiques que d'autres disciplines - comme l'anthropologie, la géographie, l'ethnologie, l'historiographie contemporaines - ont déjà parcourues avec enthousiasme. 


\section{Bibliographie}

Albrow, Martin, The Global Age: State and Society Beyond Modernity, Cambridge (UK) / Stanford (CA), Polity Press / Stanford University Press, 1996.

Appadurai, Arjun, Modernity at Large: Cultural Dimensions of Globalization, Minneapolis, University of Minnesota Press, 1996.

Bauman, Zygmunt, Globalization: The Human Consequences, New York, Columbia University Press, 1998.

Beck, Ulrich, "Remapping Social Inequalities in an Age of Climate Change: for a Cosmopolitan Renewal of Sociology ", Global Networks, vol. 10, n 2, 2010, p. 165-181.

Beck, Ulrich, Risk Society: Towards a New Modernity, London / Newbury Park / New Delhi, Sage Publications, 1992.

Beck, Ulrich, "The Cosmopolitan Condition: Why Methodological Nationalism Fails ", Theory, Culture and Society, vol. 24, $\mathrm{n}^{\text {os }} 7-8,2007$, p. 286-290.

Beck, Ulrich, The Cosmopolitan Vision, Cambridge (UK) / Malden (MA), Polity Press, 2006.

Beck, Ulrich, What Is Globalization?, Cambridge (UK) /Malden (MA), Polity Press, 2000.

Beck, Ulrich, World at Risk, Cambridge (UK) / Malden (MA), Polity Press, 2009.

Beck, Ulrich, Anthony Giddens et Scott Lash, Reflexive Modernization: Politics, Tradition and Aesthetics in the Modern Social Order, Cambridge (UK), Polity Press, 1994.

Beck, Ulrich et Edgar Grande, Cosmopolitan Europe, Cambridge (UK) / Malden (MA), Polity Press, 2007.

Beck, Ulrich et Edgar Grande, "Varieties of Second Modernity: The "Cosmopolitan Turn" in Social and Political Theory and Research", British Journal of Sociology, vol. 61, n 3, 2010, p. 409-443.

Beck, Ulrich et Natan Sznaider, "Unpacking Cosmopolitanism for the Social Sciences: A Research Agenda ", British Journal of Sociology, vol. 57, no 1, 2006, p. 1-23.

Beisheim, Marianne, Sabine Dreher, Gregor Walter, Bernhard Zangl et Michael Zürn, Im Zeitalter der Globalisierung? Thesen und Debatten zur gesellschaftlichen und politischen Denationalisierung, Baden-Baden, Nomos Verlagsgesellschaft, 1999.

Calhoun, Craig, "Beck, Asia and Second Modernity ", British Journal of Sociology, vol. 61, n 3, 2010, p. 597-619. 
Chang, Kyung-Sup, "Compressed Modernity and Its Discontents: South Korean Society in Transition ", Economy and Society, vol. 28, $\mathrm{n}^{\circ} 1$, 1999, p. 30-55.

Chang, Kyung-Sup, "The Second Modern Condition? Compressed Modernity as Internalized Reflexive Cosmopolitanism ", British Journal of Sociology, vol. 61, n 3, 2010, p. 444-464.

Ewald, François, «The Return of Descartes' Malicious Demon: An Outline of a Philosophy of Precaution ", dans Tom Baker et Jonathan Simon (dir.), Embracing Risk: The Changing Culture of Insurance and Responsibility, Chicago/Londres, University of Chicago Press, 2002, p. 273-301.

Gilroy, Paul, Black Atlantic: Modernity and Double Consciousness, Cambridge (MA), Harvard University Press, 1993.

Gilroy, Paul, «Planetarity and Cosmopolitics », British Journal of Sociology, vol. $61, n^{\circ} 3,2010$, p. 620-626.

Goldthorpe, John H, "Globalisation and Social Class ", West European Politics, vol. 25, n 3, 2002, p. 1-28.

Grande, Edgar, "Vom Nationalstaat zum transnationalen Politikregime - Staatliche Steuerungsfähigkeit im Zeitalter der Globalisierung », dans Ulrich Beck et Christoph Lau (dir.), Entgrenzung und Entscheidung. Was ist neu an der Theorie reflexiver Modernisierung?, Frankfurt am Main, Suhrkamp, 2004, p. 384-401.

Grande, Edgar, Markus König, Patrick Pfister et Paul Sterzel, « Globalization, Political Transnationalization, and the Future of the Nation-State - A Comparison of Transnational Policy Regimes ", dans S.A. Schirm (dir.), Globalization: State of the Art and Perspectives, Londres/New York, Routledge, 2007, p. 98-121.

Grande, Edgar et Louis W. Pauly (dir.), Complex Sovereignty: Reconstituting Political Authority in the Twenty-first Century, Toronto / Buffalo / Londres, University of Toronto Press, 2005.

Held, David, Anthony McGrew, David Goldblatt et Jonathan Perraton, Global Transformations: Politics, Economics and Culture, Cambridge (UK) / Stanford (CA), Polity Press / Stanford University Press, 1999. Hopkins, Anthony G. (dir.), Globalization in World History, New York, W. W. Norton, 2002.

Kant, Immanuel, "Idea for a Universal History with a Cosmopolitan Purpose ", dans Perpetual Peace and Other Essays on Politics, History and Morals, Indianapolis, Hackett, 1983, p. 29-39.

Kant, Immanuel, «Toward Perpetual Peace: A Philosophical Project », dans Practical Philosophy, Cambridge, Cambridge University Press, 1996, 
p. 311-351.

Kasperson, Jeanne X. et Roger F. Kasperson, The Social Contours of Risk, 2 volumes, Londres / Sterling (VA), Earthscan, 2005.

Katzenstein, Peter J., A World of Regions: Asia and Europe in the American Imperium, Ithaca, Cornell University Press, 2005.

Khagram, Sanjeev et Peggy Levitt, Peggy (dir.), The Transnational Studies Reader: Intersections and Innovations, Londres/New York, Routledge, 2008.

Kunelius, Risto, Elisabeth Eide, Oliver Hahn et Roland Schroeder (dir.), Reading the Mohammed Cartoons Controversy: An International Analysis of Press Discourses on Free Speech and Political Spin, Bochum/Freiburg, Projektverlag, 2007.

Kurasawa, Fuyuki, The Work of Global Justice: Human Rights as Practices, Cambridge, Cambridge University Press, 2007.

Levy, Daniel, "Recursive Cosmopolitization: Argentina and the Global Human Rights Regime ", British Journal of Sociology, vol. 61, n 3, 2010, p. 579-596.

Levy, Daniel et Natan Sznaider, Human Rights and Memory, University Park (PA), Penn State University Press, 2010.

Magris, Claudio, Danube: A Sentimental Journey from the Source to the Black Sea, Londres, Harvill Press, 1999.

Maharaj, Sarat, "Small Change of the Universal: Beyond Modernity? ", British Journal of Sociology, vol. 61, no 3, 2010, p. 565-578.

Pries, Ludger (dir.) Rethinking Transnationalism: The Meso-Link of Organizations, Abingdon (Oxon) / New York (NY), Routledge, 2008.

Randeria, Shalini, Local Refractions of Global Governance: Legal Plurality, International Institutions, the Post-Colonial State and NGOs in India, Habilitation, Free University of Berlin, 2001.

Sassen, Saskia, « Globalization or Denationalization? », Review of International Political Economy, vol. 10, n 1, 2003, p. 1-22.

Sassen, Saskia, Territory, Authority, Rights: From Medieval to Global Assemblages, Princeton, Princeton University Press, 2006.

Sassen, Saskia, The Global City: New York, London, Tokyo, Princeton, Princeton University Press, 1991.

Schiller, Nina Glick, "A Global Perspective on Migration and Development ", Social Analysis, vol. 53, n 3, 2009, p. 14-37.

Schlögel, Karl, Im Raume lesen wir die Zeit: Über Zivilisationsgeschichte und Geopolitik, München, Carl Hanser Verlag, 2003. 
Sznaider, Natan, "Rewriting the Persian Letters ", British Journal of Sociology, vol. 61, n 3, 2010, p. 627-633.

Thelen, David, "The Nation and beyond: Transnational Perspectives on United States History ", Journal of American History, vol. 86, n 3, 1999, p. 965-75.

Tsing, Anna, "Supply Chains and the Human Condition ", Rethinking Marxism, vol. 21, n 2, 2009, p. 148-76.

Tyfield, David et John Urry, "Cosmopolitan China? Lessons from International Collaboration in Low-Carbon Innovation ", British Journal of Sociology, vol. 60, n 4, 2009, p. 793-812.

Tyrrell, Ian, "Making Nations / Making States: American Historians in the Context of Empire ", Journal of American History, vol. 86, $\mathrm{n}^{\circ} 3$, 1999, p. 1015-1044.

Vertovec, Steven (dir.), Transnationalism, Londres, Routledge, 2009.

Wallerstein, Immanuel, The Modern World-System, Volume I, Capitalist Agriculture and the Origins of the European World-Economy in the Sixteenth Century, New York/Londres, Academic Press, 1974.

Wallerstein, Immanuel, The Modern World-System, Volume II, Mercantilism and the Consolidation of the European World-Economy, 1600-1750, New York, Academic Press, 1980.

Wallerstein, Immanuel, The Modern World-System, Volume III, The Second Great Expansion of the Capitalist World-Economy, 1730-1840s, San Diego, Academic Press, 1989.

Weber, Max, «Die protestantische Ethik und der Geist des Kapitalismus », dans Gesammelte Aufsätze zur Religionssoziologie, vol. 1, Tübingen, J. C. B. Mohr, 1988.

Weiß, Anja, "Vergleiche jenseits des Nationalstaats. Methodologischer Kosmopolitismus in der soziologischen Forschung über hochqualifizierte Migration ", Soziale Welt, vol. 61, nos 3-4, 2010, p. 295-311.

Zhang, Joy Yueyue, "The Cosmopolitization of Science: Experience from Chinese Stem Cell Scientists ", Soziale Welt, vol. 61, nos 3-4, 2010, p. $257-276$. 